\title{
Quantitative Evaluation of Signal Intensity of Magnetic Resonance Images in Optic Neuritis
}

\author{
Tadao Hanawa ${ }^{1}$ and Atsushi Mizota ${ }^{*}, 2$ \\ ${ }^{I}$ Department of Ophthalmology, Chiba Aoba Municipal Hospital, 1273-2 Aoba-cho, Chuoku, Chiba 260-0852, Japan \\ ${ }^{2}$ Department of Ophthalmology, Juntendo University Urayasu Hospital, 2-1-1 Tomioka, Urayasu, 279-0021, Japan
}

\begin{abstract}
We have evaluated the signal intensity of magnetic resonance (MR) images of the optic nerve quantitatively in 25 patients with unilateral acute optic neuritis $(\mathrm{ON})$. MR imaging was performed with a 1.5 Tesla unit before treatment within 2 weeks after the onset of ON. Four coronal fat-suppressed T2-weighted images were obtained at 5, 10, 15, and 20 $\mathrm{mm}$ behind the eye. The ratio of the signal intensity of the MR images from the optic nerve to that of the white matter of the frontal lobe was calculated and we compared the signal intensity ratio of the affected eyes to the fellow healthy eyes. For statistical analysis paired t-test was used. At all 4 sections, the mean signal intensity ratio of the affected eyes is statistically significant higher than fellow eyes. The 11 patients showed optic disc swelling in the affected eyes and in all these 11 eyes had a higher signal intensity at $5 \mathrm{~mm}$ behind the eye compared with the fellow eye. From our present results, we cannot refer to the sensitivity of our method, because we did not use our present methods to other diseases. But with this method we think inter-image and inter-observer variability must reduce. Further studies are required about the sensitivity and the relation between the pathological condition of optic nerve and the signal intensity ratio.
\end{abstract}

\section{INTRODUCTION}

The usefulness of magnetic resonance (MR) imaging in the diagnosis of optic neuritis (ON) was described as early as 1987 by Johnson et al. [1] using short time inversion recovery (STIR) sequences. With the technical advancement of MRI, there have been a number of studies that have not only demonstrated the usefulness of MRI but also shown an improvement in the certainty of the diagnosis [2-9].

Generally, MR imagings of the optic nerve have been interpreted by experienced neuroradiologists qualitatively. Thus in 1998, Jackson and his co-workers [7] studied the signal intensities in comparison to that of white matter and a combination of increased signal intensity and swelling in patients with $\mathrm{ON}$ and classified findings of optic nerve into 4 grades, grade 0 to 3 . This normalization was used to remove the effects of inter-image and inter-observer variability. The purpose of their study was to examine the benefits of combined fat- and water-suppressed T2-weighted MRI for the diagnosis of optic neuritis.

Using their recommendations, we have investigated quantitatively the signal intensity of the MR images of the optic nerve at several points along the optic nerve in eyes with optic neuritis to determine the effectiveness of MRI in diagnosing and assessing optic neuritis.

\section{SUBJECTS AND METHODS}

MRI was performed on 25 patients with unilateral acute $\mathrm{ON}$ before any treatment. There were 4 men and 21 women with a mean age of $36.4 \pm 13.4$ years. All patients did not have any history of $\mathrm{ON}$ in either eyes before. The diagnosis of $\mathrm{ON}$ was made on the basis of visual loss, papillary light reflex

*Address correspondence to this author at the Department of Ophthalmology, Juntendo University Urayasu Hospital, 2-1-1 Tomioka, Urayasu, 279-0021, Japan; Fax: +81-47-355-5949;

E-mail: mizota@juntendo-urayasu.jp visual field defect, optic disc findings, and abnormal pattern visual evoked potentials (PVEP) in the affected eyes [10]. In the fellow eyes all these findings were normal. This research followed the tenets of the Declaration of Helsinki and informed consent was obtained from all patients after explanation of the nature and possible consequences of the study.

MR imaging was performed on a 1.5 Tesla unit (Signa Advantage, GE, Piscataway, NJ, USA) within 2 weeks after onset of ON. Coronal fat-suppressed T2-weighted images were obtained with 4000/100 (TR $\mathrm{msec} / \mathrm{TE} \mathrm{msec}$ ) at 4 different plane, viz., 5, 10, 15, and $20 \mathrm{~mm}$ behind the eye. In all patients, there were no abnormalities in the frontal lobe. The signal intensity was measured in a 12 to $18 \mathrm{~mm}$ area of each slice of the optic nerve and white matter of the frontal lobe with Advantage Windows (GE, GE, Piscataway, NJ, USA) (Fig. 1). The size and shape of the measured area was adjusted to the size of optic nerve in each patient. To compare the signal intensities of the optic nerve, the ratio of signal intensity of optic nerve to that of white matter of the frontal lobe was calculated.

In the present study, we compared the signal intensity ratio of the affected eyes to the fellow healthy eyes. For statistical analysis paired t-test was used.

\section{RESULTS}

The MR images of a 22-year-old patient with acute unilateral optic neuritis are shown in Fig. (2). Her visual acuity was hand motion at $30 \mathrm{~cm}$ in the right and 20/15 in the left eyes. A relative afferent papillary defect was present in the right eye. The optic disc was slightly hyperemic and swollen in the right eye and was normal in the left eye. PVEP was extinguished when the pattern was presented to the right eye and normal in the left eye. The signal intensity ratios of optic nerve to frontal lobe are shown in Table 1. The signal intensity ratio of the MR images of the optic nerve of the affected right eye was higher than that of the left fellow eye at all of the 4 points behind the eye. 


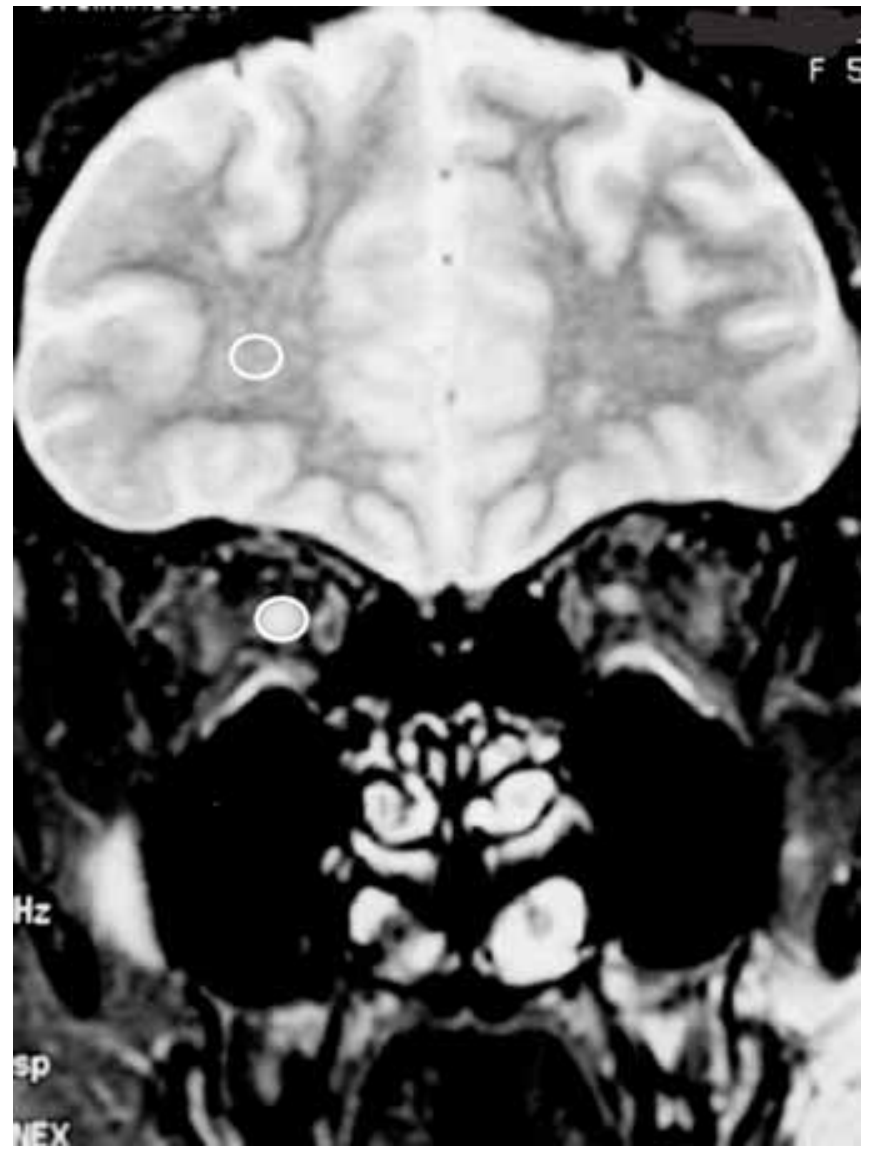

Fig. (1). Signal intensity within white circle was measured in the one coronal MR image.
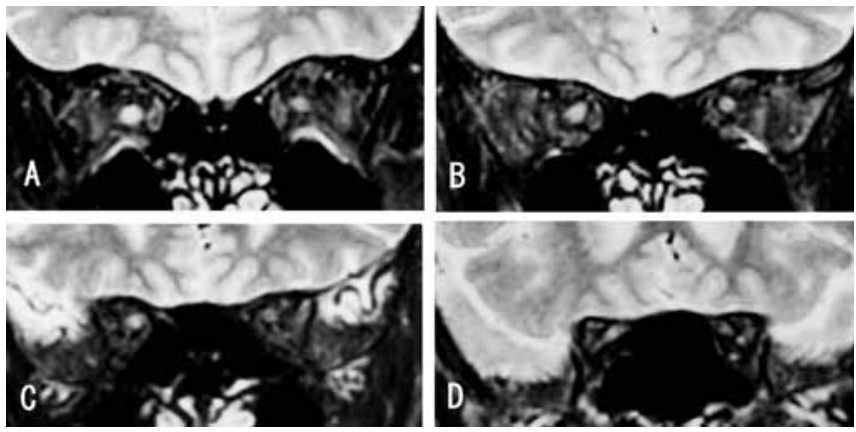

Fig. (2). Typical MR image of a case with right ON. A: $5 \mathrm{~mm}, \mathrm{~B}$ : $10 \mathrm{~mm}, \mathrm{C}: 15 \mathrm{~mm}$ and $\mathrm{D}: 20 \mathrm{~mm}$ behind eye.

Table 1. Signal Intensity Ratio (\%) in the Case Shown in Fig. (2)

\begin{tabular}{|c|c|c|c|c|}
\hline & \multicolumn{4}{|c|}{ Distance from Eye } \\
\cline { 2 - 5 } & $\mathbf{5 m m}$ & $\mathbf{1 0 m m}$ & $\mathbf{1 5 m m}$ & $\mathbf{2 0 m m}$ \\
\hline \hline Right Eye & 154.7 & 145.3 & 104.5 & 121.2 \\
\hline Left Eye & 107.6 & 85.6 & 88.1 & 88.0 \\
\hline
\end{tabular}

The mean and standard error of signal intensity ratio of the optic nerve relative to that of the frontal lobe at 4 points obtained from all of 25 patients is shown in Fig. (3). At all 4 points, the mean signal intensity ratio of the affected eyes is statistically significant higher than fellow eyes $(p=0.003$ at $5 \mathrm{~mm}, \mathrm{p}=0.001$ at $10 \mathrm{~mm}, \mathrm{p}=0.0003$ at $15 \mathrm{~mm}$ and $\mathrm{p}=0.0002$ at 20mm)

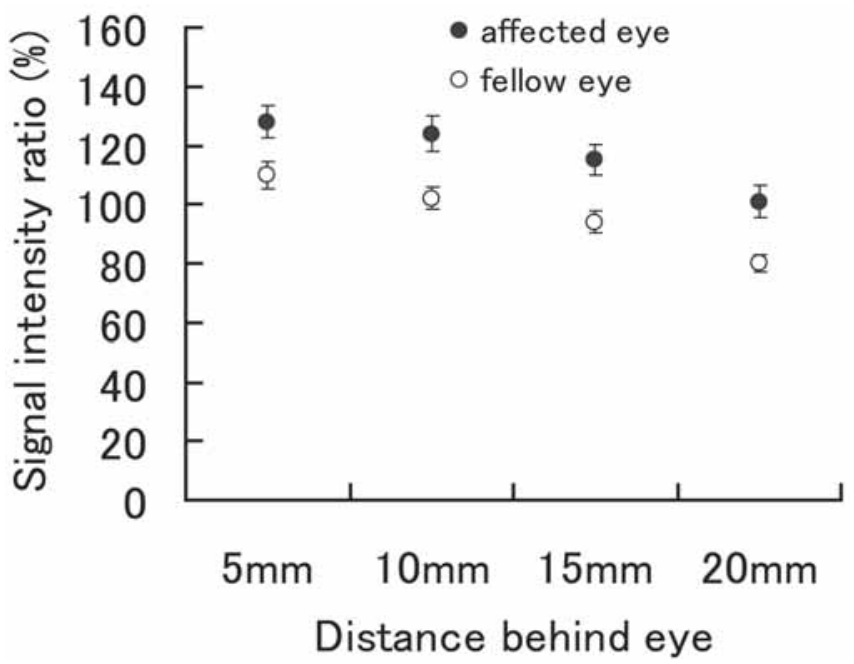

Fig. (3). Means \pm SE of the values from the 4 slices behind the eye are shown. In all parts, the ratio was significantly higher in affected eyes.

Sixteen of 25 patients with optic neuritis showed higher signal intensity at all 4 points in the affected eyes than the fellow eyes, and 23 of 25 patients showed higher mean signal intensity in the affected eye in the 4 portions. The 11 patients showed optic disc swelling in the affected eyes and in all these 11 eyes had a higher signal intensity at $5 \mathrm{~mm}$ behind the eye compared with the fellow eye.

When we define the normal range of the signal intensity ratio is within mean $\pm 2 \mathrm{SD}$ of that of fellow eyes, 12 patients had abnormally higher ratio at least one of 4 portions.

\section{DISCUSSION}

Despite the high sensitivity of MRI in detecting brain lesions in patients with multiple sclerosis [8], the imaging and the detection of abnormalities of the optic nerve have proved to be unsatisfactory because of high signal intensity of orbital fat. There have been improvements in the MRI techniques to detect optic nerve lesions in patients with acute optic neuritis since Johnson et al. [1] introduced the excellent results with the MR STIR method. Evaluations were based on signal abnormalities, such as the signal intensity [3], the enlargement of the nerve width [11], the length and the position of the lesion $[3,6,12-14]$. The precise measurement of the size and length of optic nerve lesions is considered unreliable because of the oblique placement of the optic nerve in the coronal plane.

Such unreliability of the MR images of optic nerve lesions was partly overcome by STIR [1,3], orbital fat suppression [2,7], and the T2-weighted method [6]. Among these, the T2-weighted images can detect the edema of acute lesions and the gliosis and demyelination of chronic lesions. In patients with acute $\mathrm{ON}$, signal intensity of optic nerve increases in T2-weighted MR images because of edema of optic nerve. 
The evaluation of MRI abnormalities is usually facilitated by comparisons with the normal contralateral optic nerve or with the white matter of the brain. In general, the increased signal intensity of the optic nerve is judged without any quantitative calculations. For quantitative analysis, Davies et al. [8] reported quantitative ratios of the signal intensity of the optic nerve to the other tissues, orbital fat, frontal white matter and cerebrospinal fluid, and classified into 4 grades. In the present study, we measured signal intensity of optic nerve and white matter of the frontal lobe and calculated the ratio of signal intensity of optic nerve to that of white matter in the same plane for quantitative analysis, because of the histological similarity between optic nerve and white matter. We compared the ratio of the affected eyes to the fellow eyes. Though in case of ON in multiple sclerosis there may exist the hidden $\mathrm{ON}$ in the fellow eyes, we used the fellow eyes as control because there were no sign of optic neuritis even with PVEP. In order to increase the reliability of the method, the measurements were made at 4 parts of the retrobulbar portion of the optic nerve. We found a significantly higher signal intensity in the optic nerve of eyes diagnosed with $\mathrm{ON}$ than in the optic nerve of the normal fellow eyes in all 4 parts. And 12 of 25 patients (48\%) showed statistically significantly higher signal intensity ratio at least one of 4 parts.

The Optic Neuritis Treatment Trial concluded that MR imaging has limited value in establishing the diagnosis of ON [15]. The authors recommended that MR imaging be performed for diagnostic purposes only when the clinical findings do not conform to the typical profile of optic neuritis. We agree with their conclusion because MR imaging is only one of the ways to detect abnormal lesions in the optic nerve.

\section{CONCLUSION}

We reported a method to analyze optic nerve quantitatively by MR imaging and analyzed statistically compared to the fellow eyes in patients with unilateral optic neuritis. From our present results, we cannot refer to the sensitivity of our method, because we did not apply our present methods to other diseases. But with this method we think inter-image and inter-observer variability must reduce. And if MR images of same patients are obtained in the different stage of ON, this method must be useful. Further studies are required about the sensitivity and the relation between the optic nerve pathological condition and the signal intensity ratio.

\section{REFERENCES}

[1] Johnson G, Miller DH, MacManus D, et al. STIR sequences in NMR imaging of the optic nerve. Neuroradiology 1987; 29: 238245.

[2] Larsson HB, Thomsen C, Frederiksen J, Henriksen O, Olesen J. Chemical shift selective magnetic resonance imaging of the optic nerve in patients with acute optic neuritis. Acta Radiologica 1988; 29: 629-632.

[3] Miller DH, Newton MR, van der Poel JC, et al. Magnetic resonance imaging of the optic nerve in optic neuritis. Neurology 1988; 38: $175-179$.

[4] Guy J, Mao J, Bidgood WD, Mancuso A, Quisling RG. Enhancement and demyelination of the intraorbital optic nerve. Fat suppression magnetic resonance imaging. Ophthalmology 1992; 99: 713719.

[5] Tartaro A, Onofrj M, Thomas A, Fulgente T, Delli Pizzi C, Bonomo L. Long time echo stir sequence magnetic resonance imaging of optic nerve in optic neuritis. Eur J Radiol 1995; 19: 155163.

[6] Gass A, Barker GJ, Riordan-Eva P, et al. Lesion discrimination in optic neuritis using high-resolution fat-suppresed fast spin-echo MRI. Neuroradiology 1996; 38: 317-321.

[7] Jackson A, Sheppard S, Laitt RD, Kassner A, Moriarty D. Optic neuritis: MR imaging with combined fat- and water- suppression techniques. Radiology 1998; 206: 57-63.

[8] Davies MB, Williams R, Haq N, Pelosi L, Hawkins CP. MRI of optic nerve and postchiasmal visual pathways and visual evoked potentials in secondary progressive multiple sclerosis. Neuroradiology 1998; 40: 765-770.

[9] Wilejto M, Shroff M, Buncic JR, Kennedy J, Goia C, Banwell B. The clinical features, MRI findings, and outcome of optic neuritis in children. Neurology 2006; 67: 258-262.

[10] Takasoh M, Mizota A, Adachi-Usami E. Comparetive study of pattern evoked cortical potentials in patients with ischemic optic neuropathy and optic neuritis. Acta Ophthalmol Scand 2000; 76: 407-410.

[11] Cornblath WT, Quint DJ. MRI of optic nerve enlargement in optic neuritis. Neurology 1997; 48: 821-825.

[12] Brodsky MC, Beck RW. The changing role of MR imaging in the evaluation of acute optic neuritis. Radiology 1994; 192: 22-23.

[13] Dunker S, Wiegand W. Prognotic value of magnetic resonance imaging in monosymptomatic optic neuritis. Ophthalmology 1996; 103: $1768-1773$.

[14] Kapoor R, Miller DH, Jones SJ, et al. Effect of intravenous methylprednisolone on outcome in MRI-based prognotic subgroups in acute optic neuritis. Neurology 1998; 50: 230-237.

[15] Optic Neuritis Study Group. The clinical profile of optic neuritis. Experience of the optic neuritis treatment trial. Arch Ophthalmol 1991; 109: 1673-1678. 